\title{
Cladding Effect on Hybrid Plasmonic Nanowire Cavity at Telecommunication Wavelengths
}

\author{
Pi-Ju Cheng, Chen-Ya Weng, Student Member, IEEE, Shu-Wei Chang, Member, IEEE, \\ Tzy-Rong Lin, Member, IEEE, and Chung-Hao Tien
}

\begin{abstract}
In this paper, we investigate the effect of cladding layers on a hybrid plasmonic nanowire cavity. A decent confinement factor and moderate modal loss can be achieved for lasing. Within a certain index range of the cladding layer, the fundamental hybrid plasmonic mode is well confined inside the narrow gain region and becomes the most promising lasing mode. Our results also indicate that the mirror loss can be reduced if the refractive index of the cladding layer is properly chosen. An estimated cold-cavity quality factor of 87, corresponding to a threshold gain of about $1072 \mathrm{~cm}^{-1}$, is achievable at a cavity length of $10.13 \mu \mathrm{m}$ for the lasing mode.
\end{abstract}

Index Terms-Laser resonators, plasmonic optics, semiconductor lasers, waveguides.

\section{INTRODUCTION}

$\mathbf{T}$ HE capability of plasmonic structures to confine photons in the subwavelength regime has brought new concepts into the nanophotonic technology and research [1]-[3]. Recently, there has been significant progress on nanoscale metal-related resonators aiming at an ultrasmall footprint. In spite of metal loss, subwavelength plasmonic cavities have been successfully applied to nanoscale lasers via optical or electrical pumping from low to room temperatures [4]-[15]. These plasmon lasers may have potential applications in biochemical sensing [16], imaging [17], and short-distance optical interconnects [18].

There are inevitable compromises between losses and confinements for potential plasmonic lasing modes. Although some particular plasmonic modes such as long-range surface plasmons (SPs) suffer from less loss and propagate farther, their mode profiles are often less confined than those of lossy ones. Recently, Oulton et al. proposed a hybrid plasmonic waveguide

Manuscript received July 31, 2012; revised October 30, 2012; accepted November 22, 2012. Date of publication December 3, 2012; date of current version April 25, 2013. This work was supported in part by the National Science Council, Taiwan, under Grant NSC-100-2112-M-001-002-MY2 and Grant NSC-101-2218-E-019-002, and in part by the Research Center for Applied Sciences, Academia Sinica, Taipei, Taiwan.

P.-J. Cheng and S.-W. Chang are with the Research Center for Applied Sciences, Academia Sinica, Taipei 11529, Taiwan, and also with the Department of Photonics, National Chiao Tung University, Hsinchu 30010, Taiwan.

C.-Y. Weng is with the Institute of Optoelectronic Sciences, National Taiwan Ocean University, Keelung 20224, Taiwan, and also with the Research Center for Applied Sciences, Academia Sinica, Taipei 11529, Taiwan.

T.-R. Lin is with the Department of Mechanical and Mechatronic Engineering, and Institute of Optoelectronic Sciences, National Taiwan Ocean University, Keelung 20224, Taiwan (e-mail: trlin@ntou.edu.tw).

C.-H. Tien is with the Department of Photonics, National Chiao Tung University, Hsinchu 30010, Taiwan.

Color versions of one or more of the figures in this paper are available online at http://ieeexplore.ieee.org.

Digital Object Identifier 10.1109/JSTQE.2012.2231404

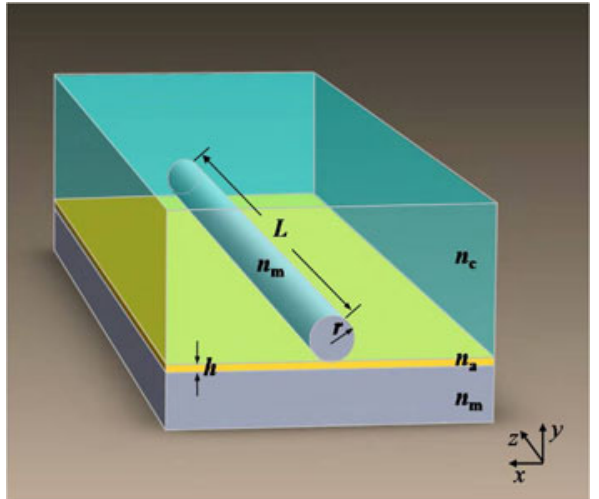

Fig. 1. Schematic diagram of a hybrid metallic nanowire cavity. The refractive indices of dielectric cladding, active layer, and metallic substrate are denoted as $n_{c}, n_{a}$, and $n_{m}$, respectively. The height $h$ of active medium, nanowire radius $r$, and cavity length $L$ are varied to investigate modal characteristics of the cavity.

composed of a high-index dielectric nanowire and metallic substrate [7], [19]. The hybrid plasmonic mode can be viewed as a coupled mode originated from the guided mode of the dielectric nanowire and the SP mode of the substrate. This mode exhibits modest attenuation and moderate field confinement. Later, $\mathrm{Hu}$ et al. proposed a hybrid plasmonic nanocavity which consists of a metallic nanowire and substrate sandwiching a dielectric gap layer [20]. Analogous to the active dielectric nanowire in the former case, the gap material in the latter plays the role of gain media, and therefore, the field leakage into the metallic nanowire should be reduced as much as possible. The experiment indicates that the cavity quality factor $(Q$ factor) is limited by the mirror loss at ends of the cavity rather than the modal loss due to the metallic nanowire and substrate. In this paper, we investigate the effect of the cladding layer on the hybrid plasmonic cavity in the presence of a metallic nanowire. Characteristics of the hybrid mode and its coupling mechanism are explored by means of the 2-D finite-element method (FEM). We also estimate the cold-cavity quality factor from the 3-D FEM. With the proper engineering of cavity geometry and selections of cladding media, $15 \%$ enhancement of the $Q$ factor can be accomplished due to improvements of the facet reflectivity.

Fig. 1 shows the schematic diagram of the hybrid plasmonic cavity. A metallic nanowire of radius $r$ is placed upon a dielectric gap layer with a height $h$. A metallic substrate is beneath the gap layer. We investigate the effect of dielectric claddings with different refractive indices $n_{c}$ around the nanowire on the formation of hybrid modes. In the absence of the dielectric gap and metallic substrate, the fundamental guided mode of the nanowire is the circularly symmetric transverse-magnetic $\left(\mathrm{TM}_{01}\right)$ mode 

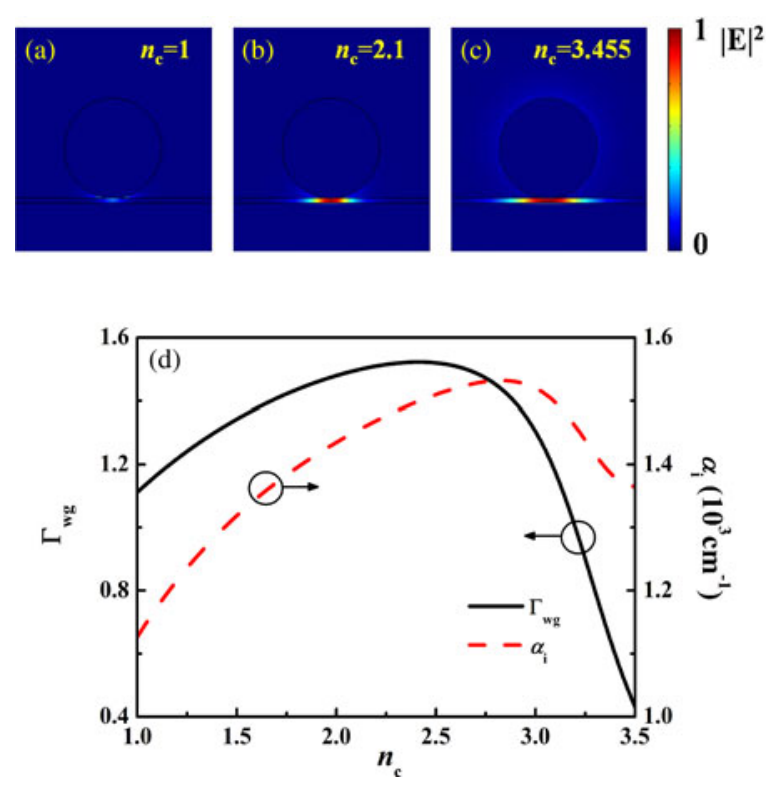

Fig. 2. Normalized field distribution of the fundamental hybrid plasmonic mode of the structure with three cladding refractive indices of (a) ambience (vacuum), (b) dielectric $\left(\mathrm{Si}_{3} \mathrm{~N}_{4}\right)$, and (c) high-index dielectric (Si). Cladding index has a significant impact on the modal profiles and confinements. (d) Waveguide confinement factor and modal loss as a function of the cladding index.

without the frequency cutoff. When this mode couples to the plasmonic mode of the planar structure composed of the dielectric gap and metallic substrate [21], the resulted hybrid mode is transversely confined in an ultrasmall area inside the dielectric gap. It is expected that the wire radius $r$ influences the dispersion relation of metallic waveguide modes, and the gap height $h$ affects the coupling strength between the modes of nanowire and planar structure [19]. In addition, we will show that the profile and propagation characteristics of this hybrid mode are also sensitive to the cladding refractive index.

\section{MOdAl CHARACTERISTICS IN HYBRID PlASMONIC WAVEGUIDE}

We numerically solve the guided modes of the hybrid plasmonic waveguide in Fig. 1 using the eigenvalue solver of the commercial software COMSOL [22]. The wavelength $\lambda$ is set to $1.55 \mu \mathrm{m}$, targeting at telecommunication applications. In the calculations, the refractive indices of metal (silver) $n_{m}$ and active region (polymethyl methacrylate, PMMA) $n_{a}$ are set to $0.16+11.09 \mathrm{i}$ [23] and 1.5 [24], [25], respectively. Three cladding refractive indices $n_{c}$ of 1 (ambience), 2.1 (silicon nitride), and 3.455 (silicon) are considered. The wire radius $r$ and gap height $h$ are set to 100 and $10 \mathrm{~nm}$, respectively, unless otherwise mentioned. In Fig. 2, the mode profiles corresponding to aforementioned settings are illustrated. The well-confined SP modes of the planar structure are strongly coupled to the $\mathrm{TM}_{01}$ mode sustained at the wire circumference through the evanescent tail of the field. Highly bright spots localized near the contact of the wire and dielectric gap, which are similar to those reported in bowtie structures [26], are observable in the square magnitude $|\mathbf{E}|^{2}$ (normalized to unity at the maximum) of the transverse mode profile in Fig. 2. Note that in Fig. 2(a), the en- ergy maximum occurs at the bottom of the nanowire rather than inside the gap as $n_{c}<n_{a}$. If the cladding index is close to that of the active dielectric $\left(n_{c} \approx n_{a}\right)$, the hybrid plasmonic mode is confined well inside the gap layer and beneath the nanowire, as shown in Fig. 2(b). This case resembles that of dielectric nanowires on the similar planar structure [7], [27]. On the other hand, with a high-index cladding $\left(n_{c}>n_{a}\right)$, the fundamental SP mode of the substrate may spread into the cladding and overlap with the mode of metallic nanowire, as indicated in Fig. 2(c). This hybrid plasmonic mode has a relatively lower portion of the field inside the dielectric gap region when compared to that in Fig. 2(b). The spread of the field in the dielectric gap and its leakage around the nanowire are also more remarkable in this case. In other words, for the given radius $r$ and height $h$ in these calculations, the hybrid plasmonic mode in Fig. 2(c) is the characteristic of both the $\mathrm{TM}_{01}$ mode of the metallic nanowire and the SP mode of the substrate. From these calculations, the modal energy density distribution is quite sensitive to the variation of the cladding refractive index $n_{c}$.

Among these three cases, the hybrid mode in Fig. 2(b) exhibits the most uniform and strong confinement inside the active gap region. Its mode profile has the best overlap with the gap layer where the optical gain is present. Therefore, adopting the mode in Fig. 2(b) as the lasing mode of an ultrasmall plasmonic laser seems promising. On the other hand, the extremely localized mode in Fig. 2(a) has the lowest modal loss because its energy distribution inside the lossy metal is the smallest among those of the three modes. To understand the field evolution of this hybrid mode as $n_{c}$ varies, we show its waveguide confinement factor $\Gamma_{\mathrm{wg}}$ and modal loss $\alpha_{i}$ as a function of $n_{c}$ in Fig. 2(d). The waveguide confinement factor $\Gamma_{\mathrm{wg}}$, an indicator of how well the mode overlaps with the gain medium, is defined as [28]

$$
\Gamma_{\mathrm{wg}}=\frac{n_{a}}{2 \eta_{0} P_{z}} \int_{A_{\mathrm{a}}} d \boldsymbol{\rho}|\mathbf{E}(\boldsymbol{\rho})|^{2}
$$

where $P_{z}$ is the power flow in the propagation direction, $A_{a}$ is the region of the active dielectric gap, $n_{a}$ is the refractive index of gain medium, and $\eta_{0}$ is the intrinsic impedance. This confinement factor is proportional to the ratio between the square magnitude of the mode in the active region and its power flow in the propagation direction. The numerator and denominator of the ratio are cross-sectional integrations of different physical quantities, and therefore, $\Gamma_{\mathrm{wg}}$ may be larger than unity [28]-[30]. On the other hand, the modal loss $\alpha_{i}$ is related to the imaginary part of $k_{z}$ as $2 \operatorname{Im}\left[k_{z}\right]$ and usually becomes larger if the field is distributed more inside lossy regions. In Fig. 2(d), maximum of $\Gamma_{\mathrm{wg}}$ occurs at $n_{c}=2.42$ and degrades mildly at the small- $n_{c}$ side until $n_{c}$ is lowered to 2 . This trend indicates that the field of the hybrid mode overlaps well with the active region in this range of $n_{c}$ and favors the choice of silicon nitride $\left(n_{c}=2.1\right)$, which is commonly available in laboratories, as the cladding. On the other hand, the modal loss $\alpha_{i}$ exhibits a similar trend to that of $\Gamma_{\mathrm{wg}}$. Since it is expected that a low modal loss can lower the lasing threshold, there is a tradeoff in the choice of $n_{c}$. In order to come up with a proper choice of the modes in Fig. 2(a)(c), we need more information to estimate the performances at different $n_{c}$.

In Fig. 3, we show the effective index $n_{\text {eff }}$ of fundamental modes as a function of the cladding refractive index for 


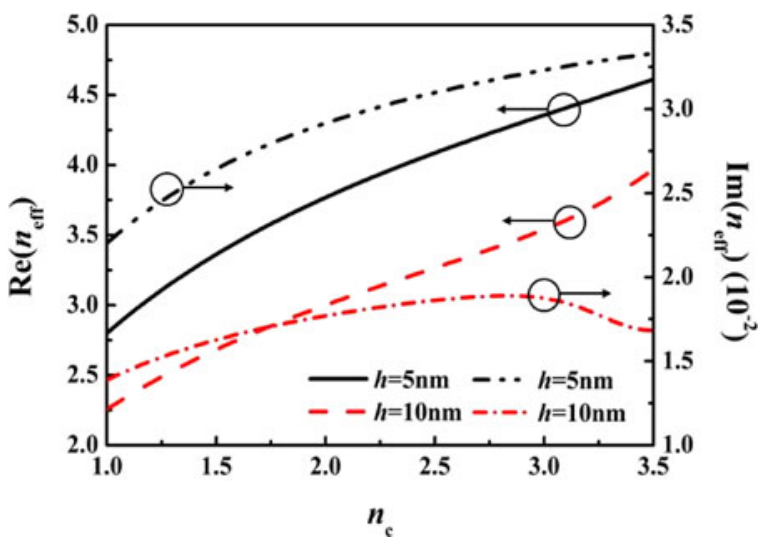

Fig. 3. Effective index $n_{\mathrm{eff}}$ of the fundamental hybrid plasmonic mode as a function of the cladding refractive index $n_{c}$ for gap heights $h=5$ and $10 \mathrm{~nm}$.
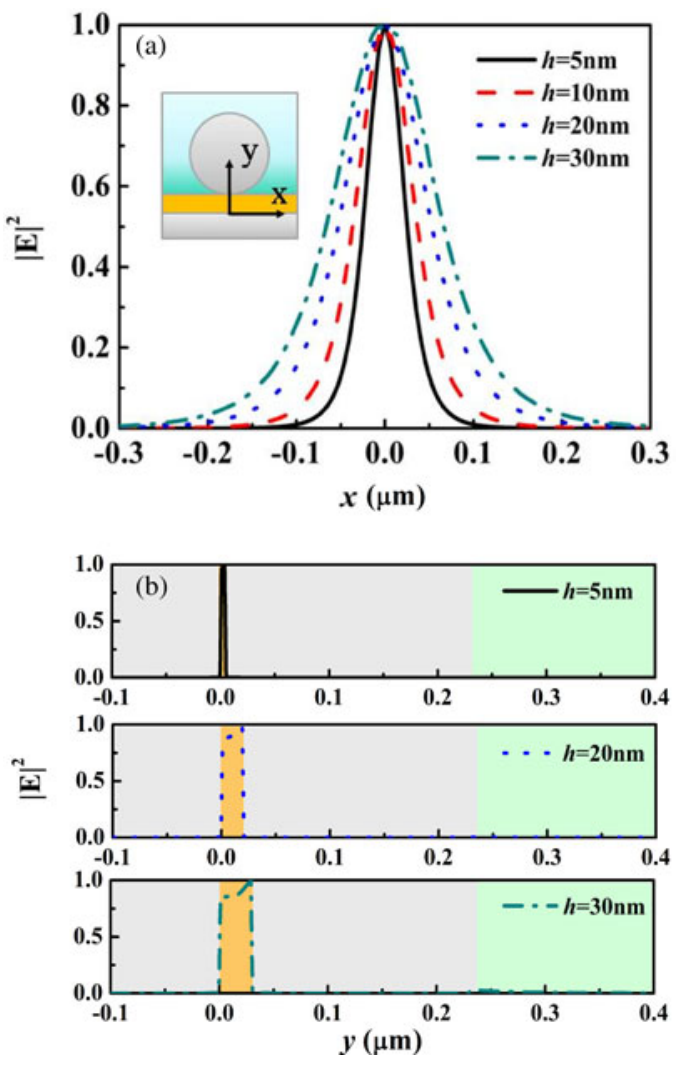

Fig. 4. Normalized field distribution in the horizontal $(x)$ and vertical $(y)$ directions. (a) Horizontal distribution at the middle of the dielectric gap. The distribution size increases with height $h$. (b) Vertical distribution at the center of the cavity. The characteristic of the $\mathrm{TM}_{01}$ mode of the metallic nanowire emerges at $y \approx 0.25 \mu \mathrm{m}$ when $h=30 \mathrm{~nm}$.

different gap heights $h$. The effective index $n_{\text {eff }}$ is defined as the ratio between the propagation constant $k_{z}$ and that $k_{0}$ in the free space. The real part of the effective index is higher than refractive indices of the cladding $n_{c}$ and dielectric gap $n_{a}$, which is the characteristic of surface-like waves. The real part $\operatorname{Re}\left[n_{\text {eff }}\right]$ increases with the cladding index $n_{c}$, but the difference between $\operatorname{Re}\left[n_{\mathrm{eff}}\right]$ and $n_{c}$ diminishes in the large- $n_{c}$ limit because the mode turns from a well-confined one in the gap into a less-confined coupled mode, as can be observed from Fig. 2(a)-(c). In addition, the dependence of the imaginary part
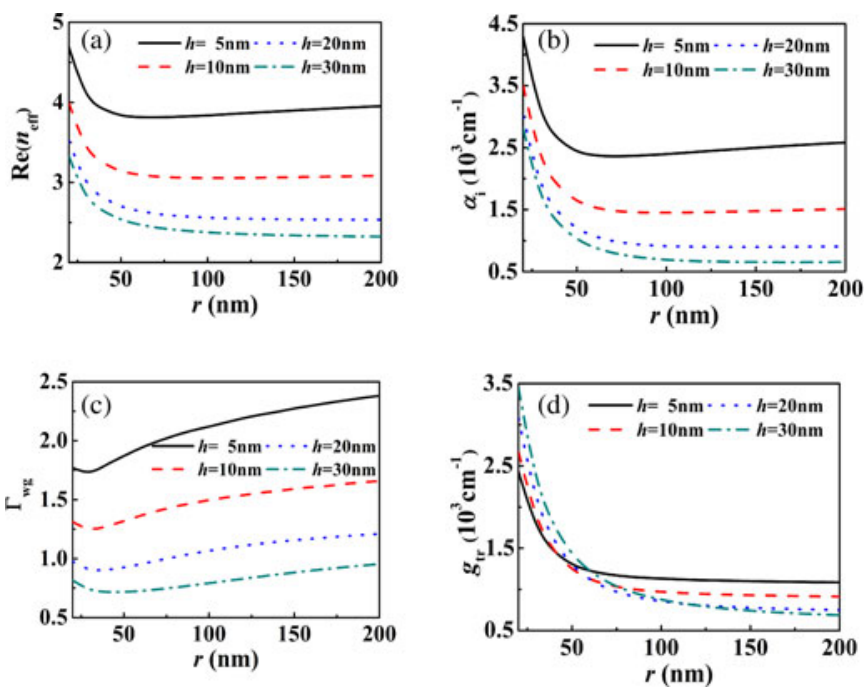

Fig. 5. (a) Real part of the effective index $\operatorname{Re}\left[n_{\text {eff }}\right]$, (b) modal loss $\alpha_{i}$, (c) waveguide confinement factor $\Gamma_{\mathrm{wg}}$, and (d) transparency threshold gain $g_{\mathrm{tr}}$ of the fundamental hybrid mode versus wire radius $r$ for different gap heights $h$.

$\operatorname{Im}\left[n_{\text {eff }}\right]$ on the cladding index $n_{c}$ indicates the degree of overlap between the mode profile and metal regions, in particular, the cross section of the metallic nanowire. To understand the subwavelength confinement in more detail, in Fig. 4, we show the normalized filed distribution $|\mathbf{E}|^{2}$ along specific directions for different gap heights. The square magnitudes along the horizontal direction ( $x$-axis) at the middle of the dielectric gap are depicted in Fig. 4(a). The localization in this direction originates from the coupling between SP modes of the planar structure and finite-sized $\mathrm{TM}_{01}$ mode of the metallic nanowire. The width of the hybrid plasmonic mode is similar to that in the case of dielectric nanowires [19] and increases with the gap height $h$, reflecting the weaker coupling between two types of modes. For the distribution along the vertical direction (y-axis) at the center of the cavity shown in Fig. 4(b), the hybrid mode is well confined. When the gap height $h$ is $30 \mathrm{~nm}$, the characteristic of the $\mathrm{TM}_{01}$ mode of the nanowire starts to emerge on the profile of the hybrid mode in this direction. A small but finite portion of field distribution on top of the nanowire $(y \approx 0.25 \mu \mathrm{m})$ can be observed.

We also show real parts of the effective index $\operatorname{Re}\left[n_{\text {eff }}\right]$, modal loss $\alpha_{i}$, waveguide confinement factors $\Gamma_{\mathrm{wg}}$, and transparency threshold gains $g_{\mathrm{tr}}$ of the fundamental hybrid mode as a function of the wire radius $r$ for different gap heights $h$ in Fig. 5(a)-(d), respectively. The cladding index $n_{c}$ is fixed at 2.1 in these calculations. For laser applications, the transparency gain $g_{\mathrm{tr}}$ is defined as the gain at which the mode can propagate through the waveguide without attenuation and is expressed as $g_{\mathrm{tr}}=\alpha_{i} / \Gamma_{\mathrm{wg}}$. This gain coefficient does not include the mirror loss $\alpha_{m}$ and is smaller than the real threshold gain $g_{\text {th }}$ of the laser. In contrast to the fundamental hybrid mode in the waveguide structure based on the dielectric nanowire [19], the fundamental mode here does not exhibit a frequency cutoff. As shown in Fig. 5(a), at the small gap height $h$, the enhanced coupling strength between the modes of the metallic nanowire and planar structure significantly increases the real part of the effective index 
$\operatorname{Re}\left[n_{\text {eff }}\right]$. From Fig. 5(b), the modal loss of the hybrid mode rapidly increases as the wire radius $r$ decreases below $100 \mathrm{~nm}$. The increase of the modal loss can be inferred from that of the $\mathrm{TM}_{01}$ mode of the metallic nanowire and the corresponding mode distribution. As the radius $r$ shrinks from 100 to $50 \mathrm{~nm}$, the modal loss $\alpha_{i}$ of the $\mathrm{TM}_{01}$ mode of the nanowire is tripled, which is caused by the more heavily weighted distribution of the mode inside the nanowire at the smaller radius. Since the hybrid mode results from the coupling between two types of modes, the less weighted distribution outside the nanowire also leads to the decrease of the waveguide confinement factor $\Gamma_{\mathrm{w} g}$ in Fig. 5(c) before the wire radius $r$ drops to $50 \mathrm{~nm}$. At the even smaller wire radius comparable to the gap height $h$, the size of the horizontal distribution inside the gap slightly rebounds. This phenomenon is due to the slight enhancement of the distribution weight inside the active dielectric gap and increases the waveguide confinement factor $\Gamma_{\mathrm{wg}}$. The overall transparency gain $g_{\mathrm{tr}}$ weakly depends on the wire radius $r$ for $r>50 \mathrm{~nm}$. For $r<50 \mathrm{~nm}$, the remarkable increase of the modal loss due to the metal dissipation raises the transparency gain $g_{\mathrm{tr}}$, and the threshold gain $g_{\mathrm{th}}$ is enhanced accordingly.

\section{DESIGN FOR A PlASMONIC LASER}

We characterize the threshold gain $g_{\mathrm{th}}$ and $Q$ factor by means of Fabry-Perot (FP) formula. The work by $\mathrm{Hu}$ et al. [20] validates the assumption of FP cavity for the hybrid plasmonic mode under the ambience condition $\left(n_{c}=1\right)$. The lasing threshold depends on the cavity length $L$ and facet reflectivity $R$. We evaluate the reflectivity $R$ by numerically solving the reflected optical field with the aid of 3-D FEM. In order to reduce the influence of unwanted reflections, we adopt the boundary condition of perfect matched layers (PMLs). In the calculation, an incident wave solely consisting of the target hybrid plasmonic mode propagates inside the cavity toward the reflection facet. The incident field is subsequently transmitted, scattered, and reflected. Only a portion of the reflected field is coupled back into the same guided mode of the incident wave while the remaining is coupled to other guided and leaky ones. This phenomenon results in a complicated interference pattern. To obtain the reflectivity $R$ of target modes, the numerical results are postprocessed with the orthogonality theorem of waveguide modes [31]. The reflectivity $R$ of the fundamental mode can be as high as 0.858 at $n_{c}=$ 2.1. For the low-index cladding of $n_{c}=1$ (high-index cladding of $n_{c}=3.455$ ), the mode profile is quite concentrated in a sharp region (leaky toward the free space of cladding) and leads to the lower reflections back into the incident mode. The reflectivities are 0.848 and 0.794 for the two cases, respectively. These results are quite different from the simple estimations based on the Fresnel formula $R=\left|\left(n_{\text {eff }}-1\right) /\left(n_{\text {eff }}+1\right)\right|^{2}$ because the plane-wave approximation is inapplicable for SP hybrid modes considered here (for example, at $n_{c}=2.1$, the Fresnel formula leads to $R=0.560)$.

The threshold gain $g_{\mathrm{th}}$ is the required gain for lasing and can be written as [32]

$$
g_{\mathrm{th}}=\frac{1}{\Gamma_{\mathrm{wg}}}\left[\alpha_{i}+\frac{1}{L} \ln \left(\frac{1}{R}\right)\right] .
$$
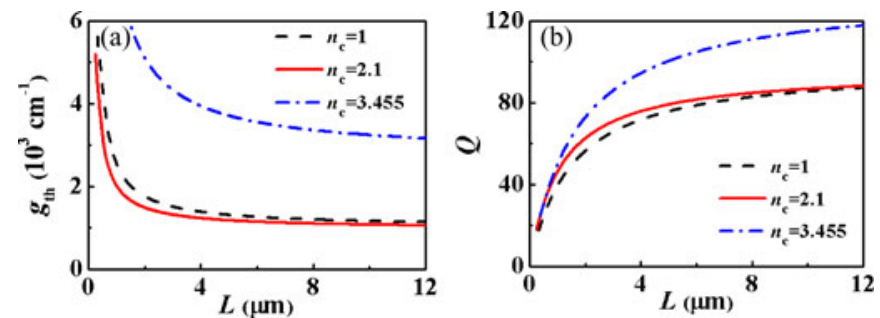

Fig. 6. (a) Threshold gain $g_{\mathrm{th}}$ and (b) $Q$ factor of the FP mode, regardless of the formation of standing waves, as a function of the cavity length $L$ for different cladding indices $n_{c}$. An increase of $15 \%$ with proper choices of the cladding index $n_{c}$ is achievable around $L=1.6-1.8 \mu \mathrm{m}$.
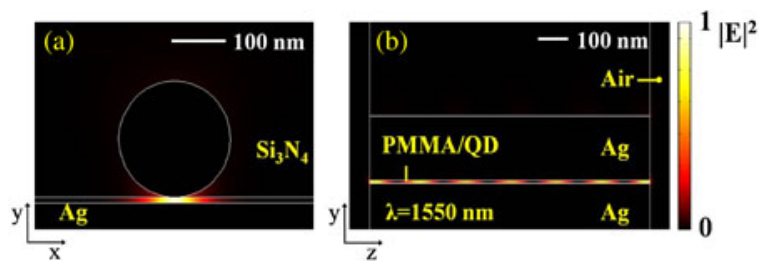

Fig. 7. Resonance patterns of the cavity mode $(L=1.55 \mu \mathrm{m})$ at the resonance wavelength of $1.55 \mu \mathrm{m}$. (a) Subwavelength confinement on the transverse cross section can be observed in the dielectric gap. (b) Standing-wave pattern along the FP oscillation axis is also present on the $y-z$ cross section.

On the other hand, the $Q$ factor is indicative of how long a photon is present in a cavity before vanishing and can be expressed as

$$
\frac{1}{Q_{\mathrm{FP}}}=\frac{v_{g, z}}{\omega}\left[\alpha_{i}+\frac{1}{L} \ln \left(\frac{1}{R}\right)\right]
$$

where $v_{g, z} \approx\left(\partial \operatorname{Re}\left[k_{z}\right] / \partial \omega\right)^{-1}$ is the group velocity of the guided mode along propagation direction, which is estimated from the frequency derivative of $\operatorname{Re}\left[k_{z}\right]$. Note that the frequency dispersion of the silver permittivity is included in the calculation of the propagation constant $k_{z}$ and automatically incorporated into the group velocity $v_{g, z}$ through the frequency derivative. The higher $Q$ factor means that the device can lase with the lower pump. The threshold gain and $Q$ factor as a function of cavity length $L$ for $n_{c}=1,2.1$, and 3.455, regardless of the formation of standing waves at $1.55 \mu \mathrm{m}$, are shown in Fig. 6(a) and (b), respectively. For $L=1.55 \mu \mathrm{m}$ at the resonance wavelength of $1.55 \mu \mathrm{m}$ $\left(n_{c}=2.1\right)$, we carry out the 3 -D FEM calculation and show the normalized field profile in Fig. 7. The transverse profile of the mode on the cross section $(x-y)$ of the cavity is depicted in Fig. 7(a), and the standing-wave pattern along the longitudinal direction is shown in Fig. 7(b). The more significant portion of the power shall be transmitted into the air region at the smaller cavity length, which is reflected on the small $Q$ factor of about 60. This indicates that the improvement of mirror designs will be necessary for the better plasmon feedback. At a cavity length $L \approx 1.6-1.8 \mu \mathrm{m}$, for instance, the difference between $Q$ factors at two cladding indices $n_{c}=1$ and $n_{c}=2.1$ is significant. At the FP resonance, the $Q$ factor at $n_{c}=2.1$ is improved by $15 \%$ when compared to that under the ambience condition at $n_{c}=1$ ( $L=1.69 \mu \mathrm{m}$ ) mainly due to the higher reflectivity at $n_{c}=2.1$, even though the cavity length at $n_{c}=1$ is actually longer. In either case, however, the intense pumping is required to overcome the high threshold gains. In order to meet a threshold gain attainable by, for example, colloidal quantum dots with optical pumping at telecommunication wavelengths [24], [25], we may 
increase the cavity length $L$ to $10.13 \mu \mathrm{m}$, at which an FP resonance is reached, for the case of $n_{c}=2.1$. With this cavity length, the $Q$ factor is enhanced to 87, and the threshold gain drops to $1072 \mathrm{~cm}^{-1}$, which makes the lasing action easier.

\section{CONCLUSION}

We have analyzed a hybrid plasmonic FP cavity containing a metallic nanowire. The coupling between the $\mathrm{TM}_{01}$ mode of the metallic nanowire and the SP mode of the planar structure is explored. The proper choice of the cladding layer brings about a uniform field distribution and decent confinement inside the active region for the fundamental plasmonic hybrid mode. For metallic nanowires with radius $r$ smaller than $50 \mathrm{~nm}$, the threshold gains rise sharply. Using 3-D FEM, we numerically extract the reflectivity of the fundamental hybrid plasmonic mode and obtain the optical field inside the FP cavity. The $Q$ factor of the cavity for $L$ around $1.6-1.8 \mu \mathrm{m}$ is improved by $15 \%$ if $n_{c}$ is varied from 1 to 2.1 . At the even smaller cavity length, the better plasmon feedback is required to reduce the mirror loss.

\section{ACKNOWLEDGMENT}

The authors would like to thank Prof. S. L. Chuang at the Department of Electrical and Computer Engineering, University of Illinois at Urbana-Champaign, for many insightful discussions, and Y.-C. Ku at the Department of Mechanical and Mechatronic Engineering, National Taiwan Ocean University, for his technical assistance.

\section{REFERENCES}

[1] J. A. Schuller, E. S. Barnard, W. Cai, Y. C. Jun, J. S. White, and M. L. Brongersma, "Plasmonics for extreme light concentration and manipulation," Nat. Mater., vol. 9, no. 4, pp. 193-204, Apr. 2010

[2] M. T. Hill, "Status and prospects for metallic and plasmonic nano-lasers," J. Opt. Soc. Amer., B, vol. 27, no. 11, pp. B36-B44, Nov. 2010.

[3] R. M. Ma, R. F. Oulton, V. J. Sorger, and X. Zhang, "Plasmonlasers: Coherent light source at molecular scales," Laser Photon. Rev., pp. 1-12, Feb. 2012.

[4] M. T. Hill, Y. S. Oei, B. Smalbrugge, Y. Zhu, T. d. Vries, P. J. van Veldhoven, F. W. M. van Otten, T. J. Eijkemans, J. P. Turkiewicz, H. de Waardt, E. J. Geluk, S. H. Kwon, Y. H. Lee, R. Nötzel, and M. K. Smit, "Lasing in metallic-coated nanocavities," Nat. Photon., vol. 1, no. 10, pp. 589-594, Oct. 2007.

[5] M. T. Hill, M. Marell, E. S. P. Leong, B. Smalbrugge, Y. Zhu, M. Sun, P. J. van Veldhoven, E. J. Geluk, F. Karouta, Y. S. Oei, R. Nötzel, C. Z. Ning, and M. K. Smit, "Lasing in metal-insulator-metal subwavelength plasmonic waveguides," Opt. Express, vol. 17, no. 13, pp. 11107-11112, Jun. 2009.

[6] M. A. Noginov, G. Zhu, A. M. Belgrave, R. Bakker, V. M. Shalaev, E. E. Narimanov, S. Stout, E. Herz, T. S. Suteewong, and U. Wiesner, "Demonstration of a spaser-based nanolaser," Nature, vol. 460, no. 7259, pp. 1110-1112, Aug. 2009.

[7] R. F. Oulton, V. J. Sorger, T. Zentgraf, R. M. Ma, C. Cladden, L. Dai, G. Bartal, and X. Zhang, "Plasmon lasers at deep subwavelength scale," Nature, vol. 461, no. 7264, pp. 629-632, Oct. 2009.

[8] C. Y. Lu, S. W. Chang, S. L. Chuang, T. D. Germann, and D. Bimberg, "Metal-cavity surface-emitting microlaser at room temperature," Appl. Phys. Lett., vol. 96, no. 25, pp. 251101-1-251101-3, Jun. 2010.

[9] S. H. Kwon, J. H. Kang, C. Seassal, S. K. Kim, P. Regreny, Y. H. Lee, C. M. Lieber, and H. G. Park, "Subwavelength plasmonic lasing from a semiconductor nanodisk with silver nanopan cavity," Nano Lett., vol. 10, no. 9, pp. 3679-3683, Sep. 2010

[10] R. M. Ma, R. F. Oulton, V. J. Sorger, G. Bartal, and X. Zhang, "Roomtemperature sub-diffraction-limit plasmon laser by total internal reflection," Nat. Mater., vol. 10, no. 2, pp. 110-113, Feb. 2011.

[11] R. A. Flynn, C. S. Kim, I. Vurgaftman, M. Kim, J. R. Meyer, A. J. Mäkinen, K. Bussmann, L. Cheng, F. S. Choa, and J. P. Long, "A room-temperature semiconductor spaser operating near $1.5 \mu \mathrm{m}$," Opt Express, vol. 19, no. 9, pp. 8954-8961, Apr. 2011.

[12] M. J. H. Marell, B. Smalbrugge, E. Jan Geluk, P. J. Veldhoven, B. Barcones, B. Koopmans, R. Nötzel, M. K. Smit, and M. T. Hill, "Plasmonic distributed feedback lasers at telecommunications wavelengths," Opt. Express, vol. 19, no. 16, pp. 15109-15118, Aug. 2011.

[13] A. M. Lakhani, M. K. Kim, E. K. Lau, and M. C. Wu, "Plasmonic crystal defect nanolaser," Opt. Express, vol. 19, no. 19, pp. 18237-18245, Sep. 2011.

[14] C. Y. Wu, C. T. Kuo, C. Y. Wang, C. L. He, M. H. Lin, H. Ah, and S. Gwo, "Plasmonic green nanolaser based on a metal-oxide-semiconductor structure," Nano Lett., vol. 11, no. 10, pp. 4256-4260, Oct. 2011.

[15] K. J. Russell, T. L. Liu, S. Chi, and E. L. Hu, "Large spontaneous emission enhancement in plasmonic nanocavities," Nat. Photon., vol. 6, no. 7 pp. 459-462, Jul. 2012.

[16] M. Loncar, A. Scherer, and Y. Qiu, "Photonic crystal laser sources for chemical detection," Appl. Phys. Lett., vol. 82, no. 26, pp. 4648-4650 Jun. 2003.

[17] Y. Nakayama, P. J. Pauzauskie, A. Radenovic, R. M. Onorato, R. J. Saykally, J. Liphardt, and P. Yang, "Tunable nanowire nonlinear optical probe," Nature, vol. 447, no. 7148, pp. 1098-1101, Jun. 2007.

[18] R. G. Beausoleil, P. J. Kuekes, G. S. Snider, S. Y. Wang, and R. S. Williams, "Nanoelectronic and nanophotonic interconnect," Proc. IEEE, vol. 96, no. 2, pp. 230-247, Feb. 2008.

[19] R. F. Oulton, V. J. Sorger, D. A. Genov, D. F. P. Pile, and X. Zhang, "A hybrid plasmonic waveguide for subwavelength confinement and longrange propagation," Nat. Photon., vol. 2, no. 8, pp. 496-500, Aug. 2008.

[20] K. J. Russell and E. L. Hu, "Gap-mode plasmonic nanocavity," Appl. Phys. Lett., vol. 97, no. 16, pp. 163115-1-163115-3, Oct. 2010.

[21] M. Z. Alam, J. Meier, J. S. Aitchison, and M. Mojahedi, "Propagation characteristics of hybrid modes supported by metal-low-high index waveguides and bends," Opt. Express, vol. 18, no. 12, pp. 12971-12979, Jun. 2010.

[22] COMSOL version 4.3, http://www.comsol.com, May 2012.

[23] P. B. Johnson and R. W. Christy, "Optical constants of the noble metals," Phys. Rev. B, vol. 6, no. 12, pp. 4370-4379, Dec. 1972.

[24] J. Grandidier, G. C. des Francs, S. Massenot, A. Bouheilier, L. Markey, J. C. Weeber, C. Finot, and A. Dereux, "Gain-assisted propagation in a plasmonic waveguide at telecom wavelength," Nano Lett., vol. 9, no. 8, pp. 2935-2939, Aug. 2009.

[25] D. Dai, Y. Shi, S. He, L. Wosinski, and L. Thylen, "Gain enhancement in a hybrid plasmonic nano-waveguide with a low-index or high-index gain medium," Opt. Express, vol. 19, no. 14, pp. 12925-12936, Jul. 2011.

[26] T. R. Lin, S. W. Chang, S. L. Chuang, Z. Zhang, and P. J. Schuck, "Coating effect on optical resonance of plasmonic nanobowtie antenna," Appl. Phys. Lett., vol. 97, no. 6, pp. 063106-1-063106-3, Aug. 2010.

[27] L. Zhu, "Modal properties of hybrid plasmonic waveguides for nanolaser applications," IEEE Photon. Technol. Lett., vol. 22, no. 8, pp. 535-537, Apr. 2010.

[28] S. W. Chang and S. L. Chuang, "Fundamental formulation for plasmonic nanolasers," IEEE J. Quantum Electron., vol. 45, no. 8, pp. 1004-1023, Aug. 2009.

[29] T. D. Visser, H. Blok, B. Demeulenaere, and D. Lenstra, "Confinement factors and gain in optical amplifiers," IEEE J. Quantum Electron., vol. 33 , no. 10, pp. 1763-1766, Oct. 1997.

[30] A. V. Maslov and C. Z. Ning, "Modal gain in a semiconductor nanowire laser with anisotropic bandstructure," IEEE J. Quantum Electron., vol. 40 , no. 10, pp. 1389-1397, Oct. 2004.

[31] A. Yariv and P. Yeh, Optical Waves in Crystals: Propagation and Control of Laser Radiation. Hoboken, NJ: Wiley, 1997, pp. 405-502.

[32] S. W. Chang, T. R. Lin, and S. L. Chuang, "Theory of plasmonic FabryPerot nanolasers," Opt. Express, vol. 18, no. 14, pp. 15039-15053, Jul. 2010.

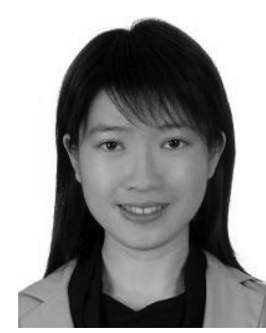

Pi-Ju Cheng is currently working toward the Ph.D degree at the Department of Photonics, National Chiao Tung University, Hsinchu, Taiwan.

Since 2011, she has also been a Student Research Assistant in the Research Center for Applied Sciences, Academia Sinica, Taipei, Taiwan. Her current research interests include plasmonic nanolasers and chiral optics. 


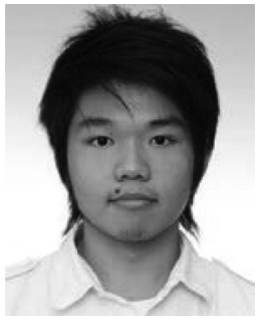

Chen-Ya Weng (S'12) received the B.S. degree in mechanical engineering from National Taiwan Ocean University, Keelung, Taiwan in 2012, where he is currently working toward the Master's degree at the Institute of Optoelectronic Sciences.

He joined the Research Center for Applied Sciences, Academia Sinica, Taipei, Taiwan as a Student Research Assistant in 2012. His research interest is in the area of plasmonics and semiconductor nanolasers.

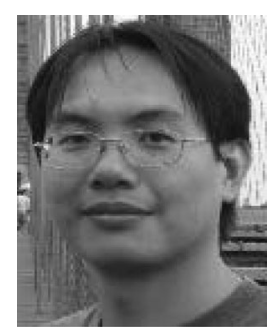

Shu-Wei Chang (M'09) received the B.S. degree in electrical engineering from National Taiwan University, Taipei, Taiwan, in 1999, and the M.S. and Ph.D. degrees from the Department of Electrical and Computer Engineering, University of Illinois at UrbanaChampaign, Urbana, in 2003 and 2006, respectively.

From 2008 to 2010, he was a Postdoctoral Associate at the Department of Electrical and Computer Engineering, University of Illinois at UrbanaChampaign. Since 2010, he has been an Assistant Research Fellow at the Research Center for Applied Sciences, Academia Sinica, Taipei. In 2011, he joined the faculties of the Department of Photonics, National Chiao Tung University, Hsinchu, Taiwan, as an Adjunct Assistant Professor. His research interest is in the area of fundamental and applied physics of semiconductor photonics including tunnelinginjection quantum-dot-quantum-well coupled system, slow and fast light in

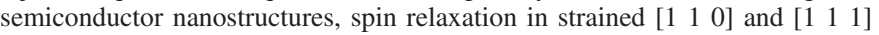
semiconductor quantum wells, group-IV direct-bandgap semiconductor lasers, active and passive plasmonic devices, semiconductor nanolasers, applications of metamaterials, both chiral and nonchiral, to semiconductor active devices, and computational schemes for both reciprocal and nonreciprocal cavities.

Dr. Chang is a member of the Optical Society of America. He received the John Bardeen Memorial Graduate Award from the Department of Electrical and Computer Engineering, University of Illinois at Urbana-Champaign, in 2006.

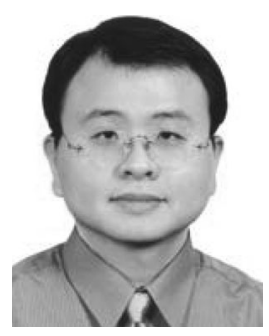

Tzy-Rong Lin (M'12) received the Ph.D. degree in applied mechanics from National Taiwan University, Taipei, Taiwan, in 2006

He was a Postdoctoral Fellow at the Institute of Applied Mechanics, National Taiwan University, from 2006 to 2007, and the Department of Electrical and Computer Engineering, University of Illinois at Urbana-Champaign, from 2007 to 2009, respectively. He has been an Assistant Professor in the Department of Mechanical and Mechatronic Engineering, National Taiwan Ocean University, Keelung, Taiwan, since 2009. He joined the faculties of the Institute of Optoelectronic Sciences at the same university, as an Adjunct Assistant Professor, in 2010. His current research interest is in the area of plasmonics and semiconductor nanolasers, and mechanical and optoelectronic coupling in semiconductor nanostructures including strained semiconductor quantum-well and quantum-dot lasers, and optomechanical devices.

Dr. Lin is a member of the Optical Society of America and the American Society of Mechanical Engineers. He received the Yen Tjing Ling Award from the Yen Tjing Ling Industrial Development Foundation in 2007.

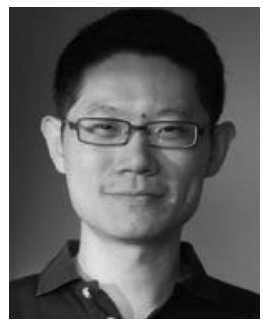

Chung-Hao Tien received the B.S. degree in communication engineering and the Ph.D. degree in electro-optical engineering from National Chiao Tung University (NCTU), Hsinchu, Taiwan, in 1997 and 2003, respectively.

After working as a Research Assistant at the University of Arizona (2001) and a Postdoctoral Fellow at Carnegie Mellon University (2003-2004), he joined NCTU as an Assistant Professor in the Department of Photonics. His research work was in the area of play, and lighting optics. computational imaging, free-form nonimaging, dis-

Dr. Tien is currently a member of the Phi Tau Phi Honor Society, the Optical Society of America, the International Society for Optical Engineers, and the Society for Information Display. 